\title{
Total Throughput Maximization of Two-Way Relay Networks Using Af Cooperative Protocol
}

\author{
Bondar V.S. ${ }^{1}$ and Sudhir S. Kanade ${ }^{2}$ \\ ${ }^{1}$ Department of electronics and Telecommunication Engineering, TPCT'S College Of Engineering, Osmanabad \\ ${ }^{2}$ Head of electronics and telecommunication Department,TPCT'S College of Engineering, Osmanabad
}

\begin{abstract}
This describes the wireless relay network where multiple user pairs conduct bidirectional communications via multiple relays based on orthogonal frequency-division multiplexing (OFDM) transmission. The joint optimization subcarrier pairing is implemented for the subcarrier allocation and relay selection. A graph based approach, is used to solve the problem optimally in polynomial time by transforming it into a maximum weighted bipartite matching (MWBM) problem. Total throughput against transmit power per node and the number of relay nodes is evaluated through which the performance of the system is analyzed for different number of subcarriers.
\end{abstract}

Keywords: - Maximum weighted bipartite matching (MWBM), orthogonal frequency - division multiplexing (OFDM), Amplify \& Forword (AF).

\section{INTRODUCTION}

The scope of the project lies in maximizing the system total throughput by optimally coordinating the relay and subcarrier assignment among the multiple pairs of two-way users. The joint optimization problem of subcarrier pairing based subcarrier assignment and relay selection for multiple two-way users is considered as a combinatorial optimization problem. Hence graph based approach is implemented to establish the equivalence between the proposed problem and a maximum weighted bipartite matching (MWBM) problem. Then the problem is solved by the corresponding graph based algorithm optimally in polynomial time. The goal is to maximize the weighted sum rate of the system by jointly optimizing subcarrier pairing and power allocation on each subcarrier in each hop. The weighting of the rates is to take into account the fact that different subcarriers may carry signals for different services. Both total and individual power constraints for the source and the relay are investigated. For the situations where the relay does not transmit on some subcarriers because doing so does not improve the weighted sum rate, we further allow the source to transmit new messages on these idle subcarriers. The channel estimation is perfect as the channel between different links experience independent fading.OFDM is very easy and efficient in dealing with multi-path and Robust again narrow-band interference As OFDM-based network supports multi relay-multi pairs of source node to conduct bidirectional communications, it maximizes the throughput optimally. There are various applications where this approach can be used such as Multihop cellular networks, Wireless media communication, Radio transmission. In AF, the relay simply amplifies the source signal linearly whereas ,in DF, the relay fully decodes, re-encodes and retransmits the source code word. Subcarrier is assigned from source to relay link and relay to destination link. In this project we are maximizing the total throughput of the system by increasing number of subcarrier.After increasing the number of subcarrier we can conclude that performance is better with more number of user.Here we have compaired performance of the system with 32 and 16 subcariers.But increase in subcarrier introduces system's power loss. InThe remainder of the paper is organized as follows. Section II introduces the literature survey. Problem formulation is detailed in Section III. Section IV provides the details of system module.

Section V provides simulations to verify the effectiveness of the algorithm. Finally, we conclude the paper in Section VI.

\section{LITERATURE SURVEY}

In AF, the relay simply amplifies the source signal linearly whereas in DF, the relay fully decodes, reencodes and retransmits the source code word. Subcarrier is assigned from source to relay link and relay to destination link. Relay assignment is investigated thoroughly in [1] and [2]. However, both the papers assume the same subcarrier for both the links in the relay based system. From [3] and [4], it is inferred that a better performance can be achieved if both the links assume different subcarriers which can be optimally paired .OFDM is employed for transmission over time dispersive channels in the two-way relay network (TWRN), where two source terminals exchange their information through a relay terminal using AF and DF relaying schemes. We propose a two-phase protocol for the channel estimation, which is compatible with the two-phase data transmission scheme. In the first phase, the two source terminals send their individual training sequences 
concurrently to the relay nodes. In order to avoid inter-pair interference, each user pair occupies non overlapping subcarriers. The intrapair interference will be treated as back-propagated self interference and cancelled perfectly after two-way relaying .In the second phase, the signal is modified based on therelay strategy selected and broadcasted to the destinations.Compared with the existing works in [1]-[7], our problem involves two major technical challenges. The first one isthe subcarrier pairing and assignment. Though the optimal subcarrier pairing has been found for one-way relaying such as [3]-[5], only heuristic subcarrier pairing methods are available for two-way relaying [6], [7]. In addition, the problem is more involved in the multi-user scenario since subcarriers should not only be carefully paired for each two-way link but also be assigned adaptively for different users. The second challenge lies in the fact that subcarrier pairing and relay selection are tightly coupled, i.e. different relay selections lead to different subcarrier pairing and assignment, and vice versa. In this work we do not pursue power allocation for simplicity. It is known that power allocation can bring significant improvement in relay networks when all source and relay nodes are subject to a total power constraint [3]. However, the gain brought by power allocation is very limited in OFDM-based relay networks if each transmitting node is subject to an individual peak power constraint [3], [5], [6]. In the considered system model, all nodes are subject to their own individual peak power constraints and therefore, the transmit power is assumed to be uniformly distributed across all subcarriers.

\section{PROBLEM FORMULATION}

The subcarriers are $\mathrm{n}$ and $\mathrm{n}$ ' in the first and second phase respectively. If the user pair $k$ is assigned with subcarrier $n$ and sends signals to relay $r$ in the first phase, the relay $r$ then broadcasts the amplified received signals on subcarrier $n^{\prime}$ in the second phase,

The achievable sum rate is given by,

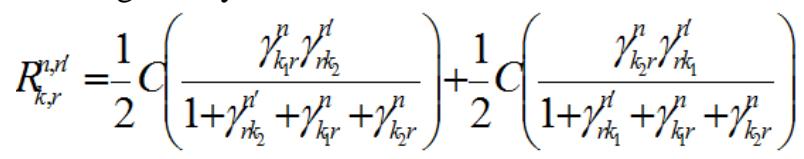

Where $C(x)=\log (1+x)$ and $\gamma_{i j}^{n}$ denotes the instantaneous signal-noise ratio (SNR) from node $\mathrm{i}$ to node $\mathrm{j}$ over subcarrier $\mathrm{n}$, assuming that all the nodes have the unit noise variance.

Let us consider the set of binary variables $\rho_{k, r}^{n, n^{\prime}}=\{0,1\}$ for all k, r,n,n' Where $\rho_{k, r}^{n, n^{\prime}}=1$ means that subcarrier $\mathrm{n}$ in the first phase is paired with subcarrier $\mathrm{n}$ ' in the second phase assisted by relay $\mathrm{r}$ for user pair $\mathrm{k}$, else $\rho_{k, r}^{n, n^{\prime}}=0$ otherwise .Hence it should satisfy the following constraints.

$$
\sum_{k \in \mathcal{K}} \sum_{r \in \mathcal{M}} \sum_{n^{\prime} \in \mathcal{N}} \rho_{k, r}^{n, n^{\prime}} \leq 1, \quad \forall n \in \mathcal{N},
$$

$$
\sum_{k \in \mathcal{K}} \sum_{r \in \mathcal{M}} \sum_{n \in \mathcal{N}} \rho_{k, r}^{n, n^{\prime}} \leq 1, \quad \forall n^{\prime} \in \mathcal{N} .
$$

Finally the objective function can be formulated as,

$$
\begin{aligned}
& \text { P1: } \quad \max \sum_{k \in \mathcal{K}} \sum_{r \in \mathcal{M}} \sum_{n \in \mathcal{N}} \sum_{n^{\prime} \in \mathcal{N}} R_{k, r}^{n, n^{\prime}} \rho_{k, r}^{n, n^{\prime}} \\
& \text { s.t. }(2),(3) .
\end{aligned}
$$

A graph based approach is proposed to solve the problem optimally in polynomial time. The problem is P1, a combinatorial optimization problem. Here the P1 is transformed to P2 which is equivalent to a maximum weighted bipartite matching (MWBM) problem. A bipartite graph is a graph whose vertices are divided into two disjoint sets so that every edge connects a vertex in one set to one in another. If the two sets of vertices have the same cardinality, then the bipartite graph is a balanced bipartite graph. A matching is a set of mutually disjoint edges, i.e., any two edges do not share a common vertex. A perfect matching is a matching that every vertex in the graph is matched. A perfect matching is the special case of matching.With this approach a pair of matched vertices is just a subcarrier pair in the MAC and BC phases, a matching implies no violating the exclusive subcarrier assignment in each phase, and the weighting process done for each edge in $\mathcal{E}$ is equivalent to finding the optimal user pair and relay for each possible subcarrier pair can be found. In this proposed system, the problem reduces to selecting the optimal user pair and relay for each subcarrier for throughput maximization, which can be optimally solved by the greedy algorithm. 
A greedy algorithm is an algorithm that follows the problem solving heuristic of making the locally optimal choice at each stage with the hope of finding a global optimum. In many problems, a greedy strategy does not in general produce an optimal solution, but nonetheless a greedy heuristic may yield locally optimal solutions that approximate a global optimal solution in a reasonable time.

\section{SYSTEM MODEL}

An OFDM-based wireless network with $K$ pairs of users and $M$ relays is shown in Fig. 1,where each user pair exchange information via the relays. Each node operates in a half-duplex mode. For simplicity, the amplify andforward (AF) two-way relay strategy is adopted.

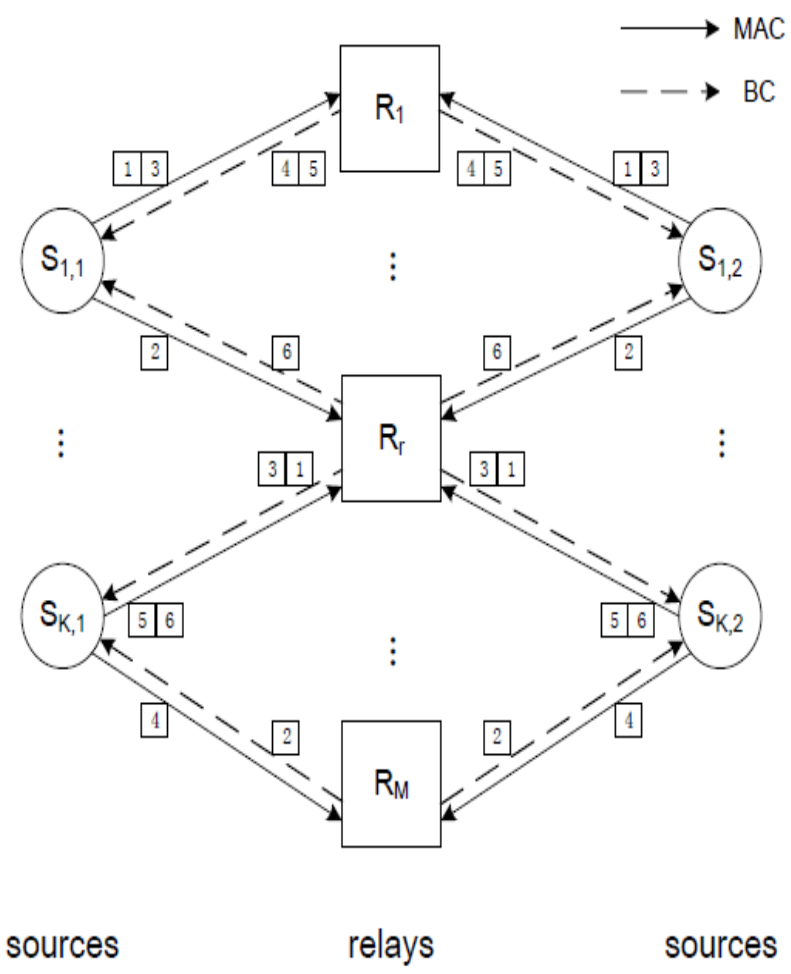

FIG .1.OFDM-based wireless network with $\mathrm{K}$ pairs of users and M relays

The wireless fading environment by large-scale path loss and shadowing, along with small-scale frequency-selective fading is considered.The channels between different links experience independent fading and the network operates in slow fading environment, so that channel estimation is perfect.A central controller is available in the network so that the centralized processing is possible. The additive white noises at all nodes are assumed to be independent circular symmetric complex Gaussian random variables and the direct communication link between the two users in each pair is neglected due to, for instance, the shadowing effects.

The two way communication takes place in two phases. The first phase is multiple-access (MAC) phase where all the $K$ pairs of users concurrently transmit signals to the relay nodes. In order to avoid inter-pair interference, each user pair occupies non-overlapping subcarriers. The intra-pair interference will be treated as back-propagated self interference and canceled perfectly after two-way relaying. In the second phase, known as broadcast (BC) phase, the relay nodes amplify the received signal and then forwards to the $2 K$ destinations. Again, each relay is operating on non overlapping subcarriers to avoid inter-relay interference.OFDMOrthogonal-frequency-division multiplexing (OFDM) employed for transmission over time-dispersive channels in the two-way relay network (TWRN), where two source terminals exchange their information through a relay terminal using the amplify-and-forward (AF) relaying scheme. We propose a two-phase training protocol for the channel estimation, which is compatible with the two-phase data transmission scheme. In the first phase, the two source terminals send their individual training sequences concurrently to the relay, while in the second phase the relay amplifies the received training sequences and broadcasts them to both source terminals. 


\section{SIMULATION RESULTS}

The simulation results can be obtained by assuming that the path loss exponent is set to 4 and the standard deviation of log-normal shadowing is set to $5.8 \mathrm{~dB}$. The Performance comparison of the proposed algorithm and the benchmark is evaluated using the greedy algorithm and proves that complexity of the proposed system is higher than the benchmark. This shows the total throughput increase when there are $\mathrm{K}=5$ user pairs and $\mathrm{M}=4$ relays in the network. And also this shows the throughput performance versus the number of relays.

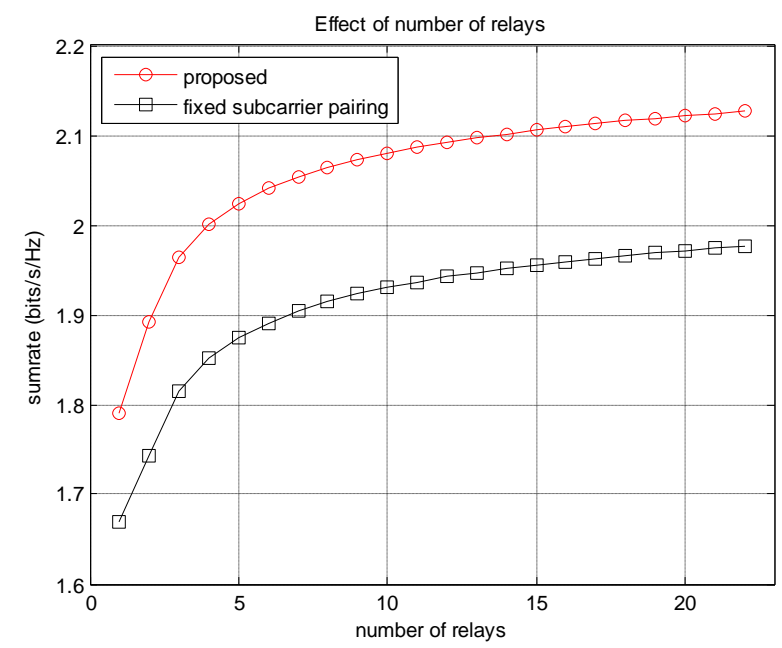

FIG.2. Effects of the number of relays, where $N=32, \quad K=5 \mathrm{M}=4$

\section{CONCLUSION}

In the proposed system, the joint optimization of subcarrier-pairing based subcarrier assignment and relay selection for multi-relay multi-pair two-way relay OFDM networks was analyzed and the problem was formulated as a combinatorial optimization problem and NP-complete. A bipartite matching approach was proposed to solve the problem optimally in polynomial time. The work assumed the amplify-and-forward based non regenerative relay strategy. Hence with the input as Delay spread is $5 \mu s$,Doppler spread and subcarrier $\mathrm{N}=32$. The output will be maximized Sum-rate (bits/Hz) .

\section{REFERENCES}

[1] G. Li and H. Liu, "Resource allocation for OFDMA relay networks with fairness constraints," IEEE J. Sel. Areas Commun., vol. 24, no. 11, pp. 2061-2069, Nov.2006.

[2] R.E. Moore, Interval analysis (Englewood Cliffs, NJ: Prentice-Hall, 1966).

[3] T. C.-Y. Ng and W. Yu, "Joint optimization of relay strategies and resource allocations in cooperative cellular networks,” IEEE J. Sel. AreasCommun., vol.25, no. 2, pp. 328-339, Feb. 2007.

[4] Hammerstrom and A. Wittneben, "Power allocation schemes for amplify-and-forward MIMO-OFDM relay links," IEEE Trans. WirelessCommun., vol. 6, no. 8, pp. 2798-2802, Aug. 2007.

[5] Y. Li, W. Wang, J. Kong, and M. Peng, "Subcarrier pairing for amplify-and-forward and decode-and forward OFDM relay links," IEEE Commun. Lett.,vol. 13, no. 1, pp. 209-211, Apr. 2009.

[6] W. Dang, M. Tao, H. Mu, and J. Huang, "Subcarrierpair based resource allocation for cooperative multirelayOFDM systems," IEEE Trans.WirelessCommun., vol. 9, no. 5, pp. 1640-1649, May 2010.

[7] C. K. Ho, R. Zhang, and Y. C. Liang, "Two-way relaying over OFDM: optimized tone permutation and power allocation," in Proc. 2008 IEEEICC, pp. 3908-3912.

[8] Y. Liu, M. Tao, B. Li, and H. Shen, "Optimization framework and graph-based approach for relay assisted bidirectional OFDMA cellular networks,"IEEETrans. Wireless Commun., vol. 9, no. 11, pp.3490-

[9] 3500, Nov. 2010.

[10] B. Rankov and A. Wittneben, "Spectral efficient protocols for half duplex fading relay channels," IEEE J. Sel. Areas Commun., vol. 25, no. 2, pp.379-389, Feb.2007.

[11] X. Zhang, A. Ghrayeb, and M. Hasna, "Network coding and relay assignment schemes for systems with multiple two-way relay channels," in Proc. 2010 IEEE ICC.

[12] X. J. Zhang and Y. Gong, "Adaptive power allocation in two-way amplify-and-forward relay networks," in Proc. 2009 IEEE ICC. 
[13] Y.-U. Jang, E.-R. Jeong, and Y. H. Lee, "A two-step approach to power allocation for OFDM signals over two-way amplify-and-forward relay, "IEEE Trans. Signal Process., vol. 58, no. 4, pp. 2426-2430, Apr. 2010.

[14] D. West, Introduction to Graph Theory. Prentice Hall, 2001.

[15] V. Erceg, L. Greenstein, S. Tjandra, S. Parkoff, A. Gupta, B. Kulic,A. Julius, and R. Jastrzab, "Anempirically based path loss modelfor wireless channels in suburban environments," IEEE J. Sel.Areas Commun., vol. 17, no. 7, pp. 1205-1211, July 